\title{
Experimental Verification of Comparability between Spin-Orbit and Spin-Diffusion Lengths
}

\author{
Yasuhiro Niimi, ${ }^{1}$, Dahai Wei, ${ }^{1}$ Hiroshi Idzuchi, ${ }^{1}$ Taro Wakamura,,${ }^{1}$ Takeo Kato, ${ }^{1}$ and YoshiChika Otani ${ }^{1,2}$ \\ ${ }^{1}$ Institute for Solid State Physics, University of Tokyo, \\ 5-1-5 Kashiwa-no-ha, Kashiwa, Chiba 277-8581, Japan \\ ${ }^{2}$ RIKEN-ASI, 2-1 Hirosawa, Wako, Saitama 351-0198, Japan
}

(Dated: January 3, 2013)

\begin{abstract}
We experimentally confirmed that the spin-orbit lengths of noble metals obtained from weak anti-localization measurements are comparable to the spin diffusion lengths determined from lateral spin valve ones. Even for metals with strong spin-orbit interactions such as Pt, we verified that the two methods gave comparable values which were much larger than those obtained from recent spin torque ferromagnetic resonance measurements. To give a further evidence for the comparability between the two length scales, we measured the disorder dependence of the spin-orbit length of copper by changing the thickness of the wire. The obtained spin-orbit length nicely follows a linear law as a function of the diffusion coefficient, clearly indicating that the Elliott-Yafet mechanism is dominant as in the case of the spin diffusion length.
\end{abstract}

PACS numbers: 73.20.Fz, 72.25.Ba, 73.63.Nm, 75.75.-c

Spin relaxation and spin dephasing are the central issues in the field of spintronics as they determine how long and far electrons can transfer the spin information 1, 2]. Owing to recent technological advancements 3 - 12], one can create the spin accumulation, i.e., the electrochemical potential difference between spin-up and down electrons at the Fermi level, $10 \sim 100$ times larger than that generated in conventional lateral spin valve devices or along edges of samples with spin Hall effects (SHEs). Such a large spin accumulation can induce a large pure spin current, the flow of spin angular momentum with no net charge current [13]. As its magnitude scales with the spin relaxation length or the spin diffusion length (SDL), the quantitative evaluation of the SDL is of importance.

Recent reports on magnetization switching at very thin ferromagnet/nonmagnet bilayer films [11, 12, 14] have triggered a heavy debate on the detailed mechanism. The first realization of the switching was reported by Miron et al. [14] who concluded that the magnetization switching originates from the Rashba effect at the ferromagnet(Co)/nonmagnet(Pt) interface. Similar measurements were also performed by Liu et al. with a $\mathrm{Co} / \mathrm{Pt}$ bilayer film [11] as well as with a $\mathrm{CoFeB} /$ Ta bilayer one [12]. They claimed that the switching is due to the perpendicularly induced spin currents via the SHE of Pt and Ta. To discuss the conversion efficiency from charge current to spin current i.e., the spin Hall ( $\mathrm{SH}$ ) angle, in their devices, they performed the spin torque induced ferromagnetic resosnance (FMR) measurements and estimated the $\mathrm{SH}$ angle of $\mathrm{Pt}$ and $\mathrm{Ta}$ to be 0.07 and 0.15 , respectively [10 12]. These SH angles, however, are quite different from those obtained from the spin absorption method (0.02 for Pt and 0.004 fo Ta) [15]. Furthermore Liu et al. [16] pointed out that the overestimation of the SDLs reported in Ref. [15] results in a large underestimation of the $\mathrm{SH}$ angles. To settle down such heavy debates, one needs another reliable way to estimate the SDL or the SH angle. In this Letter, we focus on weak anti-localization (WAL) observed in nonmagnetic metals. We first demonstrate that the spin-orbit (SO) length $L_{\mathrm{SO}}$ obtained from the WAL curve of $\mathrm{Ag}$ is comparable to the SDL $L_{\mathrm{s}}$ estimated from the lateral spin valve measurement. We then extend a similar discussion to a strong $\mathrm{SO}$ material such as Pt. In metallic systems where the elastic mean free path $l_{e}$ is much shorter than $L_{\mathrm{s}}$, the dominant spin relaxation process is the Eliott-Yafet (EY) mechanism [17]. We also confirm that the EY mechanism works very well for $L_{\mathrm{SO}}$ by changing the diffusion coefficient $D$ of $\mathrm{Cu}$ wires. This experimental fact also verifies the comparability between $L_{\mathrm{SO}}$ and $L_{\mathrm{S}}$.

We prepared two types of devices, i.e., samples for WAL measurements and those for spin injection measurements. Both samples were fabricated on a thermallyoxidized silicon substrate using electron beam lithography on polymethyl-methacrylate resist and a subsequent lift-off process. For the WAL samples, we prepared $\sim 1 \mathrm{~mm}$ long and $100 \mathrm{~nm}$ wide $\mathrm{Ag}(99.99 \%), \mathrm{Cu}$ (99.9999\%), and Pt (99.98\%) wires and performed the standard 4 -probe measurement using a ${ }^{3} \mathrm{He}$ cryostat. In order to obtain a very small WAL signal compared to the background resistance, we used a bridge circuit [18]. For the spin injection (or spin valve) measurement, we first prepared two Permalloy $\left(\mathrm{Ni}_{81} \mathrm{Fe}_{19}\right.$; hereafter Py) wires, which work as spin injector and detector. To measure $L_{\mathrm{s}}$ of a strong $\mathrm{SO}$ material such as $\mathrm{Pt}$, we inserted it in between the two Py wires. The three wires were bridged by a thicker $\mathrm{Cu}$ wire to transfer a pure spin current generated at the $\mathrm{Py} / \mathrm{Cu}$ interface. To check the reproducibility, we measured at least a few different samples on the same batch both for the WAL and spin valve measurements.

In order to clarify whether $L_{\mathrm{SO}}$ from WAL measure- 


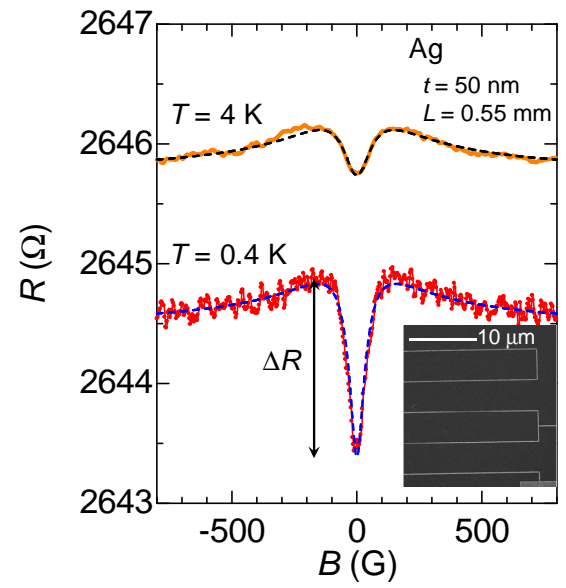

FIG. 1: (Color online) WAL curves of Ag wire measured at $T=0.4$ and $4 \mathrm{~K}$. The broken lines are the best fits of Eq. (1). The inset shows a scanning electron micrograph of the $\mathrm{Ag}$ wire.

ments is equivalent to $L_{\mathrm{s}}$ from spin valve ones, we first measure WAL curves of a weak SO material. Figure 1 shows typical WAL curves of a $\mathrm{Ag}$ wire measured at $T=0.4$ and $4 \mathrm{~K}$. Unlike a normal weak localization (WL) curve, the resistance increases with increasing the perpendicular magnetic field $B$ because of the SO interaction. With decreasing temperature, the phase coherence of electrons gets larger and the WAL peak also gets sharper. The WAL peak of quasi one-dimensional (1D) wire can be fitted by the Hikami-Larkin-Nagaoka formula [19];

$\frac{\Delta R}{R_{\infty}}=\frac{1}{\pi L} \frac{R_{\infty}}{\hbar / e^{2}}\left(\frac{\frac{3}{2}}{\sqrt{\frac{1}{L_{\varphi}^{2}}+\frac{4}{3} \frac{1}{L_{\mathrm{SO}}^{2}}+\frac{1}{3} \frac{w^{2}}{l_{B}^{4}}}}-\frac{\frac{1}{2}}{\sqrt{\frac{1}{L_{\varphi}^{2}}+\frac{1}{3} \frac{w^{2}}{l_{B}^{4}}}}\right)$

where $\Delta R, R_{\infty}, L$, and $w$ are respectively the WL correction factor, the resistance of the wire at high enough field, the length and width of the quasi-1D wire. $e, \hbar$, and $l_{B}=\sqrt{\hbar / e B}$ are respectively the electron charge, the reduced Plank constant, and the magnetic length. In Eq. (11), we have only two unknown parameters; $L_{\varphi}$ and $L_{\mathrm{SO}}$. According to the Fermi liquid theory [20], $L_{\varphi}$ does depend on temperature $\left(\propto T^{-1 / 3}\right)$, while $L_{\mathrm{SO}}$ is almost constant at low temperatures 21]. Based on this fact, we fix $L_{\mathrm{SO}}$ at both temperatures to fit the WAL curves. We obtain $L_{\varphi}=4.20$ and $1.95 \mu \mathrm{m}$ at $T=0.4$ and $4 \mathrm{~K}$, respectively, while $L_{\mathrm{SO}}=800 \mathrm{~nm}$ [22]. The two $L_{\varphi}$ values meet the Fermi liquid theory $L_{\varphi} \propto T^{-1 / 3}$. We have measured 4 different $\mathrm{Ag}$ wires on the same batch and obtained $L_{\mathrm{SO}}=760 \pm 50 \mathrm{~nm}$.

$L_{\mathrm{SO}}$ should be closely related to $L_{\mathrm{s}}$. This relation has been theoretically discussed in Ref. [1]. The SO scattering rate $1 / \tau_{\mathrm{SO}}=D / L_{\mathrm{SO}}^{2}$ includes both spin-flip and spin-conserving processes, resulting in $1 / \tau_{\mathrm{SO}}=3 /\left(2 \tau_{\uparrow \downarrow}\right)$ where $1 / \tau_{\uparrow \downarrow}$ is the spin-flip scattering rate. We also note that the spin relaxation rate $1 / \tau_{\mathrm{s}}=D / L_{\mathrm{s}}^{2}$ is twice the spin-flip scattering rate, i.e., $1 / \tau_{\mathrm{s}}=1 / \tau_{\uparrow \downarrow}+1 / \tau_{\downarrow \uparrow}$. At sufficiently low temperatures, the contribution of phonons can be neglected and one obtains

$$
L_{\mathrm{s}}=\frac{\sqrt{3}}{2} L_{\mathrm{SO}}
$$

within the EY mechanism from isotropic impurity scattering. Since $\mathrm{Ag}$ is a monovalent metal with an almost spherical Fermi surface, one can adapt Eq. (2) to convert from $L_{\mathrm{SO}}$ to $L_{\mathrm{s}}$. We thus obtain $L_{\mathrm{s}}=650 \pm 40 \mathrm{~nm}$, which is quantitatively consistent with $L_{\mathrm{s}}(\sim 600 \mathrm{~nm})$ obtained from the lateral spin valve measurements [4, 23].

Next we discuss the SDL of a strong SO material such as $\mathrm{Pt}$ which is the most standard SHE material. As mentioned in the introduction, this is one of the causes of the big debates, i.e., $L_{\mathrm{s}}=11 \mathrm{~nm}$ from the spin absorption measurements [15] and $L_{\mathrm{s}}=1.4 \mathrm{~nm}$ from the spin torque FMR measurements [16]. To solve the problem, here we perform two different measurements to obtain $L_{\mathrm{SO}}$ or $L_{\mathrm{s}}$ of Pt; WAL and spin absorption in the lateral spin valve devices. Note that the Pt wires for the WAL and spin absorption measurements were prepared at the same time. Figure 2(a) shows a typical WAL curve of Pt. The field scale is 20 times larger than that for $\mathrm{Ag}$ wires, which indicates that $L_{\mathrm{SO}}$ is much shorter. We observe maxima of the WAL curve at around \pm 0.9 T. From the best fit of Eq. (1), we obtain $L_{\mathrm{SO}}=12 \mathrm{~nm}$. We have performed similar measurements for 5 different samples and using Eq. (2) we have determined $L_{\mathrm{s}}$ of $\mathrm{Pt}$ to be $10 \pm 2 \mathrm{~nm}$ [24].

The spin absorption measurement into the $\mathrm{Pt}$ wire is shown in Fig. 2(b). For comparison, Py middle-wire devices were prepared since the SDL of Py is well-known 1) from other experiments 25]. We have performed nonlocal spin valve (NLSV) measurements with and without the middle wires [8, 9, 15]. The in-plane magnetic field $B_{\|}$is applied parallel to the two Py wires [see the inset of Fig. 2(b)]. A pure spin current generated from Py1 is absorbed perpendicularly into the middle wire because of the strong $\mathrm{SO}$ interaction of $\mathrm{Pt}$ (or Py). As shown in Fig. 2(b), the NLSV signal detected at Py2 is reduced by inserting the $\mathrm{Pt}$ or $\mathrm{Py}$ wire compared to the one without any middle wire. To extract the SDLs of $\mathrm{Pt}$ and Py, we first use the 1D analytical model based on the Takahashi-Maekawa formula [13]. In this model, the normalized NLSV signal $\Delta R_{\mathrm{S}}^{\text {with }} / \Delta R_{\mathrm{S}}^{\text {without }}$ can be expressed as follows [8, 9];

$$
\frac{\Delta R_{\mathrm{S}}^{\text {with }}}{\Delta R_{\mathrm{S}}^{\text {without }}} \approx \frac{2 R_{\mathrm{M}} \sinh \left(d / L_{\mathrm{s}}^{\mathrm{Cu}}\right)}{R_{\mathrm{Cu}}\left\{\cosh \left(d / L_{\mathrm{s}}^{\mathrm{Cu}}\right)-1\right\}+2 R_{\mathrm{M}} \sinh \left(d / L_{\mathrm{s}}^{\mathrm{Cu}}\right)}
$$

where $R_{\mathrm{Cu}}$ and $R_{\mathrm{M}}$ are the spin resistances of $\mathrm{Cu}$ and the middle wire ( $\mathrm{Pt}$ or $\mathrm{Py}$ ), respectively. The spin resistance $R_{\mathrm{X}}$ of material "X" is defined as $\rho_{\mathrm{X}} L_{\mathrm{S}}^{\mathrm{X}} /\left(1-p_{\mathrm{X}}^{2}\right) A_{\mathrm{X}}$, where $\rho_{\mathrm{X}}, L_{\mathrm{S}}^{\mathrm{X}}, p_{\mathrm{X}}$ and $A_{\mathrm{X}}$ are respectively the electrical resistivity, the SDL, the spin polarization, and the effective 


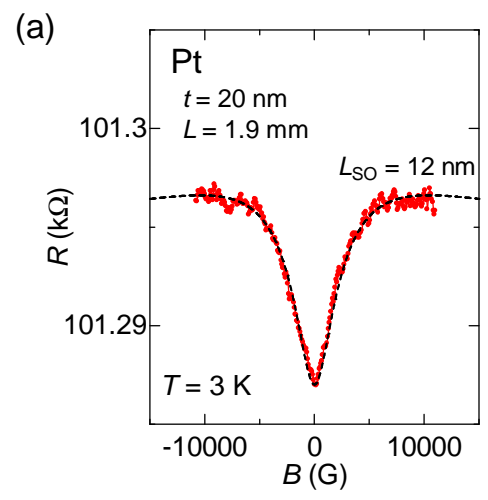

(b)

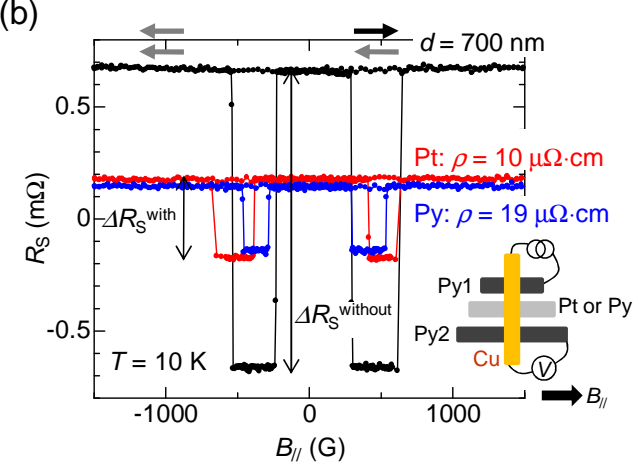

FIG. 2: (Color online) (a) WAL curve of Pt wire measured at $T=3 \mathrm{~K}$. The broken line is the best fit of Eq. (1). (b) NLSV signals with $\mathrm{Pt}\left(R_{\mathrm{S}}^{\text {with }}\right.$; red) and Py (blue) wires in between the two Py wires. As a reference signal, we plot the NLSV signal without the middle wire $\left(R_{\mathrm{S}}^{\text {without }}\right.$; black $)$. The width and thickness of the Pt or Py middle wire are $100 \mathrm{~nm}$ and $20 \mathrm{~nm}$, respectively. The other dimensions are the same as in Refs. [8] and [9]. A pair of arrows on the top indicates the magnetizations of Py1 and Py2. The inset shows the schemetic of our lateral spin valve device.

cross sectional area involved in the equations of the 1D spin diffusion model [8, 9]. $d$ is the distance between the two Py wires, in the present case $d=700 \mathrm{~nm}$. Although the spin absoprtion rate $\Delta R_{\mathrm{S}}^{\mathrm{with}} / \Delta R_{\mathrm{S}}^{\text {without }}$ is almost the same for the Pt and Py middle wires, the obtained $L_{\mathrm{s}}$ from Eq. (3) are $11( \pm 2) \mathrm{nm}$ for Pt and $5( \pm 1) \mathrm{nm}$ for Py. This is because the resistivity of $\mathrm{Pt}$ is nearly half of $\mathrm{Py}$. The SDL of Pt coincides well with that from our WAL measurement and the SDL of Py is also consistent with other experimental results 25$]$.

We have also used the three-dimensional (3D) spin diffusion model based on the Valet-Fert formalism [26] to obtain $L_{\mathrm{s}}$ of Pt. This has been done in order to refute the claim made by Liu et al. that $L_{\mathrm{s}}$ of $\mathrm{Pt}$ extracted from the 1D model might be overestimated [16]. As detailed in Ref. [9], SDLs obtained from the two methods do not differ significantly when the SDLs are comparable or smaller than the thickness of the middle wire. In fact, we have confirmed that the $3 \mathrm{D}$ analysis for the Pt middle wire gives almost the same value as the $1 \mathrm{D}$ model. We thus conclude that the SDL of Pt itself is not of the order of $1 \mathrm{~nm}$ but about $10 \mathrm{~nm}$.

The reason why $L_{\mathrm{s}}$ of $\mathrm{Pt}$ reported in Ref. 16] is much shorter than ours is that in the FMR measurement, the ferromagnet/nonmagnet bilayer is always used. In such a bilayer system, one cannot avoid the contribution from the magnetic damping effect [16, 27, 28]. As a result, the real SDL of nonmagnet can be modulated by the FMR, which results in a much shorter SDL. Thus, such a shorter SDL cannot be adapted to the case of the spin aborption method and the $\mathrm{SH}$ angle of $\mathrm{Pt}$ should be about a few percent [15], should not be enhanced up to $7 \%$ as claimed by Liu et al [16]. Recently, Kondou et al. 29] measured the $\mathrm{SH}$ angle of $\mathrm{Pt}$ using the same method as Liu et al. 10 12, 16 but they carefully studied the thickness dependence of ferromagnet and nonmagnet. They found that the symmetric part of FMR spectra, from which the $\mathrm{SH}$ angle is extracted, does depend on the thickness of ferromagnet and one should take the zero-limit to avoid any effects from the ferromagnet 29]. The extrapolated $\mathrm{SH}$ angle is 0.022 for $\mathrm{Pt}$, which is quantitatively consistent with that in Ref. [15].

To further support the comparability between $L_{\mathrm{SO}}$ and $L_{\mathrm{s}}$, we study the disorder effect on $L_{\mathrm{SO}}$. In metallic systems where $l_{e}<L_{\mathrm{s}}$, the $\mathrm{EY}$ mechanism is dominant for the spin relaxation process. If Eq. (2) is valid in metallic systems, $L_{\mathrm{SO}}$ should also follows the EY mechanism. For this purpose, we have chosen copper which has a weak SO interaction and then simply changed the thickness $t_{\mathrm{Cu}}$ of the copper wire. Figure 3(a) shows WAL curves of $20,30,80 \mathrm{~nm}$ thick $\mathrm{Cu}$ wires. It is obvious that the maximum position (triangle in the figure) due to the SO interaction shifts toward lower fields with increasing $t_{\mathrm{Cu}}$. This position corresponds to the field where $2 / L_{\mathrm{SO}}=w / l_{B}^{2}$, i.e., $B^{*}=2 \hbar /\left(e w L_{\mathrm{SO}}\right)$. From the fitting, we obtain $L_{\mathrm{SO}}=340,550,920 \mathrm{~nm}$ for $t_{\mathrm{Cu}}=20,30$, $80 \mathrm{~nm}$ respectively.

In Fig. 3(b) we plot $L_{\mathrm{SO}}$ of $\mathrm{Cu}$ as a function of $D$. Note that $D$ is determined from the Einstein relation $D=1 /\left(e^{2} \rho N\right)$ where $N$ is the density of state at the Fermi level [30]. $L_{\mathrm{SO}}$ nicely follows a linear law down to $20 \mathrm{~nm}$ thick $\mathrm{Cu}$ wires. According to the EY mechanism, $\tau_{\mathrm{s}}$ consists of the phonon and impurity contributions as follows; $1 / \tau_{\mathrm{s}}=1 / \tau_{\mathrm{s}}^{\mathrm{imp}}+1 / \tau_{\mathrm{s}}^{\mathrm{ph}}$. Since we focus on the low temperature part, we can neglect the phonon contribution 21] and concentrate on the discussion only about the spin relaxation from impurities, which makes the analysis much simpler 31]. In addition, the impurity contribution can be expressed as $\tau_{\mathrm{s}}^{\mathrm{imp}}=\tau_{e} / \varepsilon_{\mathrm{imp}}$ where $\tau_{e}$ and $\varepsilon_{\mathrm{imp}}$ are the elestic scattering time and the probability of spin-flip scattering, respectively [32]. Thus, one obtains the following equation;

$$
L_{\mathrm{SO}}=\frac{2}{\sqrt{3}} L_{\mathrm{s}}=\frac{2 D}{v_{\mathrm{F}} \sqrt{\varepsilon_{\mathrm{imp}}}}=\frac{2 l_{e}}{3 \sqrt{\varepsilon_{\mathrm{imp}}}}
$$

where $v_{\mathrm{F}}$ is the Fermi velocity. As can be seen in 
(a)
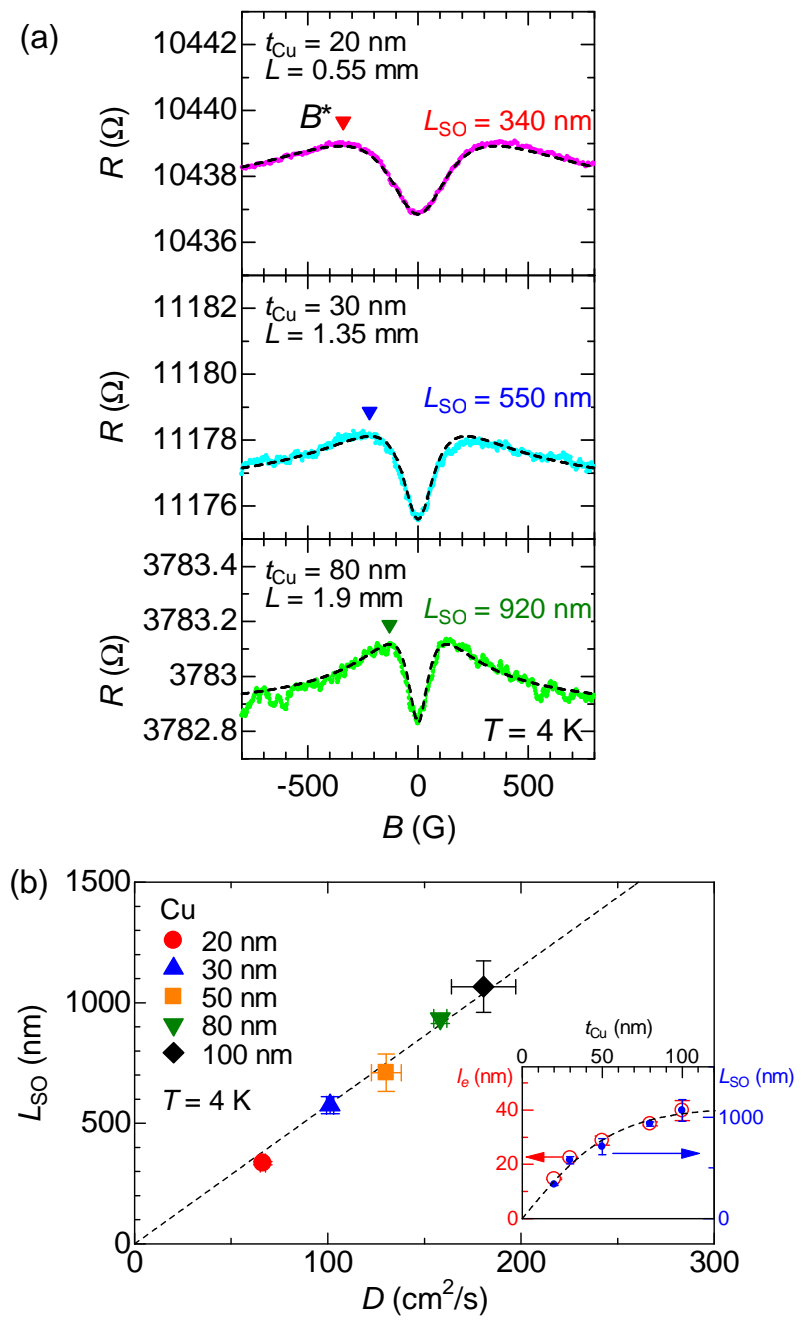

FIG. 3: (Color online) (a) WAL curves of $\mathrm{Cu}$ wires with different thicknesses $\left(t_{\mathrm{Cu}}=20,30\right.$, and $\left.80 \mathrm{~nm}\right)$ measured at $T=4 \mathrm{~K}$. The broken lines are the best fits of Eq. (11). The triangle in the figure corresponds to $B^{*}$. (b) Diffusion coefficient $D$ dependence of $L_{\mathrm{SO}}$ of $\mathrm{Cu}$ measured at $T=4 \mathrm{~K}$. The inset shows $l_{e}$ (left) and $L_{\mathrm{SO}}$ (right) as a function of $t_{\mathrm{Cu}}$. The broken line is a guide to eyes.

Fig. 3(b), the simplified EY mechanism Eq. (4) works down to at least $D \sim 60 \mathrm{~cm}^{2} / \mathrm{s}$. Although there are many experimental works to determine the SDL [4, 6, 32] in the lateral spin valve structure, the disorder dependence of the SDL has not been focused on in those reports. As far as we know, there is only one report by Bass and Pratt to mention the disorder effect on $L_{\mathrm{SO}}$ [33]. However, since they collected data from several different papers measured at different temperatures, it is not trivial to exclude the phonon contribution. Therefore, the present work is a clear experimental demonstration to verify Eq. (4). From the fitting of $L_{\mathrm{SO}}$ vs $D$ curve, we estimate $\varepsilon_{\text {imp }}$ to be $4.9 \times 10^{-4}$. This value is consistent with the ones obtained from the spin valve mesurement [32] and the
CESR measurements [34]. We also show $L_{\mathrm{SO}}$ and $l_{e}$ as a function of $t_{\mathrm{Cu}}$ on the same plot in the inset of Fig. 3(b). Both $L_{\mathrm{SO}}$ and $l_{e}$ follow the same dependency. When $t_{\mathrm{Cu}}$ is much larger than $l_{e}$, the impurity and defect contributions come mainly from the inside of the wires. With decreasing $t_{\mathrm{Cu}}, l_{e}$ is limited by $t_{\mathrm{Cu}}$. This means that the present system is perfectly diffusive and there is no specular scattering from the surface [18]. In this case, the surface scattering can be regarded as a kind of impurity or defect, and thus Eq. (4) works down to our lowest $D$.

In conclusion, we have experimentally verified the comparability between the SO lengths and the SDLs of noble metals using the WAL and spin absorption methods. This comparability works not only for weak SO materials but also for strong SO materials such as Pt. We have also studied the disorder effect on the SO lengths of $\mathrm{Cu}$ by changing the thickness of wires. The obtained SO length nicely follows a linear law as a function of $D$, which clearly verifies the EY mechanism in the present system.

We acknowledge helpful discussions with C. Bäuerle, S. Maekawa, S. Takahashi, S. Kasai and K. Kondou. We would also like to thank Y. Iye and S. Katsumoto for the use of the lithography facilities. This work was supported by KAKENHI.

* Electronic address: niimi@issp.u-tokyo.ac.jp

[1] I. Zŭtić, J. Fabian, and S. Das Sarma, Rev. Mod. Phys. 76, 323 (2004).

[2] J. Fabian and D. Das Sarma, J. Vac. Sci. Technol. B 17, 1708 (1999).

[3] T. Yang, T. Kimura, and Y. Otani, Nature Phys. 4, 851 (2008).

[4] Y. Fukuma et al., Appl. Phys. Lett. 97, 012507 (2010).

[5] Y. Fukuma et al., Nature Mater. 10, 527 (2011).

[6] Y. K. Takahashi et al., Appl. Phys. Lett. 100, 052405 (2012).

[7] T. Seki et al., Nature Mater. 7, 125 (2008).

[8] Y. Niimi et al., Phys. Rev. Lett. 106, 126601 (2011).

[9] Y. Niimi et al., Phys. Rev. Lett. 109, 156602 (2012).

[10] L. Liu et al., Phys. Rev. Lett. 106, 036601 (2011).

[11] L. Liu et al., Phys. Rev. Lett. 109, 096602 (2012).

[12] L. Liu et al., Science 336, 555 (2012).

[13] S. Takahashi and S. Maekawa, Phys. Rev. B 67, 052409 (2003); Sci. Technol. Adv. Mater. 9, 014105 (2008).

[14] M. Miron et al., Nature Mater. 9, 230 (2010); Nature (London) 476, 189 (2011).

[15] M. Morota et al., Phys. Rev. B 83, 174405 (2011).

[16] L. Liu, R. A. Buhrman, and D. C. Ralph, arXiv:1111.3702

[17] R. J. Elliott, Phys. Rev. 96, 266 (1954); Y. Yafet, in Solid State Physics, edited by F. Sitz and D. Turnbull (Academic, New York, 1963), Vol. 14.

[18] Y. Niimi et al., Phys. Rev. Lett. 102, 226801 (2009); Phys. Rev. B 81, 245306 (2010).

[19] E. Akkermans and G. Montambaux, Mesoscopic physics of electrons and photons (Cambridge University Press, 
Cambridge, 2007).

[20] B. L. Altshuler, A. G. Aronov and D. E. Khmelnitsky, J. Phys. C 15, 7367 (1982).

[21] The Debye temperature of $\mathrm{Ag}(\mathrm{Cu})$ is about 220 (320) K. In the present situation $(T \leq 4 \mathrm{~K})$, the phonon contribution to $L_{\mathrm{SO}}$ is negligibly small.

[22] F. Pierre et al., Phys. Rev. B 68, 085413 (2003).

[23] G. Mihajlović et al., Phys. Rev. Lett. 104, 237202 (2010).

[24] Strictly speaking, the prefactor in Eq. (2) would not be correct for $\mathrm{Pt}$ since $\mathrm{Pt}$ is not a simple monovalent metal. However, it should not be so different from $\sqrt{3} / 2$.

[25] S. Dubois et al., Phys. Rev. B 60, 477 (1999).

[26] T. Valet and A. Fert, Phys. Rev. B 48, 7099 (1993).

[27] S. Mizukami, Y. Ando, and T. Miyazaki, Phys. Rev. B 66, 104413 (2002).
[28] F. Fohr et al., Phys. Rev. Lett. 106, 226601 (2011).

[29] K. Kondou et al., Appl. Phys. Exp. 5, 073002 (2012).

[30] $N=2.51 \times 10^{22}$ states $/ \mathrm{eV} / \mathrm{cm}^{3}$ for $\mathrm{Cu}$.

[31] H. Idzuchi et al., Appl. Phys. Lett. 101, 022415 (2012).

[32] F. J. Jedema, A. T. Filip, and B. J. van Wees, Nature (London) 410, 345 (2001); F. J. Jedema et al., Phys. Rev. B 67, 085319 (2003).

[33] J. Bass and W. P. Pratt, J. Phys. Condes. Matter 19, 183201 (2007); Note that in this paper they assume $L_{\mathrm{SO}}=L_{\mathrm{s}}$.

[34] F. Beuneu and P. Monod, Phys. Rev. B 13, 3424 (1976); Phys. Rev. B 18, 2422 (1978); P. Monod and F. Beuneu, Phys. Rev. B 19, 911 (1979). 